\title{
Notfallsonografie des Thorax
}

Tanja Kaneko, Wolfgang Heinz

\begin{abstract}
Bei Erkrankungen im Thoraxbereich sind in Notfallsituationen bildgebende Verfahren wie CT oder Röntgen nur begrenzt einsetzbar. Sie sind meist nicht bettseitig verfügbar oder haben keine hohe Aussagekraft. Die Thoraxsonografie kann eine sinnvolle Ergänzung sein und in manchen Fällen gar die Primärbildgebung darstellen [1]. Der Beitrag zeigt, welche Anlotungen hilfreich sind und wie Sie die wichtigsten Erkrankungen rasch erkennen.
\end{abstract}

Abb.1 (a) Anlotung von anterior, z. B. bei Frage nach einem Pneumothorax im Rahmen einer FAST-Untersuchung. (b) Anlotung von lateral, z. B. bei Suche nach einem Pleuraerguss beim liegenden Patienten.
Richtige Sonde auswählen | Welche Sonde man wählt, hängt von der klinischen Fragestellung ab. Für oberflächennahe Strukturen wie Rippen und Pleura bietet sich ein Linearschallkopf mit hoher Frequenz und damit guter Auflösung im Nahbereich an. Bei modernen Geräten kann man aber auch mit einem Konvexschallkopf ein ausreichend gutes Bild erzeugen. Vorteil sind die größere Eindringtiefe und das breitere Schallfeld.

Mit einem Konvexschallkopf lassen sich Pathologien besser darstellen, die großflächig der Thoraxwand anliegen - z. B. ein größerer Pleuraerguss.

Durch die kleine Kontaktfläche eines Sektorschallkopfs gelingt ein optimaler Blick in die Tiefe von interkostal ohne störende Rippenartefakte. Voraussetzung ist, dass die darzustellenden Pathologien bis an die viszerale Pleura reichen und nicht von lufthaltiger Lunge überdeckt werden.
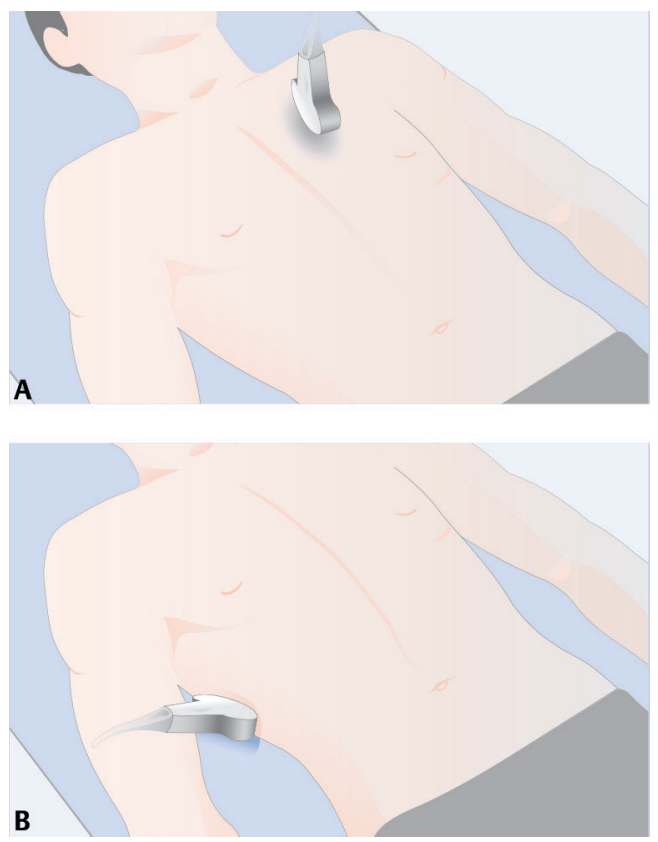

\section{Anlotungen}

Bei Pneumothorax | Für die gezielte Anlotung ist es entscheidend, die Lagerungsabhängigkeit bestimmter Pathologien zu kennen. Bei einem Pneumothorax wandert die Luft im Pleuraspalt entgegen der Schwerkraft, sofern keine Adhäsionen vorliegen. Patienten im Schockraum werden meistens in Rückenlage behandelt. Standardmäßig wird bei einer erweiterten FAST-Untersuchung („focussed assessment of sonography in trauma“) im 2. bzw. 3. Interkostalraum in der Medioklavikularlinie ( $\bullet$ Abb. 1a) angelotet. Sollte der Patient sitzen, z. B. bei einer Pleurapunktion, wird man weiter apikal nach den Zeichen des Pneumothorax suchen.

Bei Flüssigkeitsansammlungen I Im Gegensatz zum Pneumothorax sammeln sich Flüssigkeiten egal ob intrapleural oder interstitiell - durch die Schwerkraft am tiefsten Punkt. Suchen Sie also kleine Pleuraergüsse und Zeichen des interstitiellen Syndroms am besten beim sitzenden Patienten am kaudalsten Punkt im Bereich der Zwerchfellgrenze. Befindet sich der Patient in Rückenlage, sollte so dorsal wie möglich angelotet werden ( Abb. 1b).

In den abhängenden Bereichen treten bedingt durch hydrostatische Effekte mehr B-Linien auf.

Unabhängig von der Lagerung macht es Sinn, dort zu schallen, wo ein auffälliger klinischer Befund erhoben wurde oder an umschriebenen Schmerzpunkten.

\section{Normalbefund}

Lungengleiten | Von schallkopfnah nach schallkopffern sieht man die Thoraxwand mit Subkutis, Muskulatur und Rippen ( $\triangleright$ Abb. 2). Letztere weisen im nicht verknöcherten Bereich keine sonst charakteristischen dorsalen Schallauslöschungen auf, sodass dort die Pleura nicht überschattet 
wird. Die Pleura parietalis und visceralis erscheint als eine echoreiche Linie, die sich atemabhängig bewegt, so als ob Ameisen auf ihr liefen. Dieses Phänomen nennt man „Lungengleiten“.

Tipps für eine bessere Darstellung I Um das Lungengleiten besser darzustellen, sollte man

- artefaktreduzierende Geräteeinstellungen ausschalten (harmonic imaging etc.),

- Fokus bewusst unterhalb der Pleuralinie platzieren,

- Verstärkung ggf. reduzieren,

- Schallkopf bei Bedarf so anwinkeln (wippen), dass die Schallwellen tangentialer (nicht $90 \mathrm{Grad}$ ) auf die Pleuralinie treffen.

A- und B-Linien | Fernab dieser Linie spiegelt sich die Thoraxwand inklusive Pleura in den vermeintlichen Lungenraum hinein. Es entstehen horizontale Reverberationen - die A-Linien. Vereinzelt sieht man B-Linien, die als echoreiche, vertikal verlaufende Kometenschweifartefakte von der Pleura bis zum Bildende reichen.

Limitation | Im Normalzustand lässt sich die Lunge jenseits der Pleuralinie sonografisch nicht abbilden. Erst wenn eine Pathologie bis an die Pleura heranreicht und nicht von lufthaltiger Lunge überdeckt ist, entsteht ein „schallbares“ Fenster. Dies ist eine der Hauptlimitationen dieser Untersuchungstechnik.

Für die bessere Orientierung ist es hilfreich, initial quer zum Rippenverlauf zu schallen. Somit kann man Rippen- und Lungenartefakt mühelos unterscheiden.

\section{Leitsymptom Dyspnoe}

Pleuraerguss I Ein mit Dyspnoe einhergehender Pleuraerguss ist meist schon klinisch gut zu diagnostizieren. Mit Ultraschall lassen sich jedoch auch kleine Pleuraergüsse darstellen. Liegt der Patient in einer optimalen Position, kann man schon geringe Mengen (ab $5 \mathrm{ml}$ ) nachweisen. Gleichzeitig kann die Sonomorphologie des Ergusses Hinweise auf die zugrunde liegende Erkrankung geben und so wertvolle Zusatzinformationen bieten.

Lungenarterienembolie I Weniger bekannt ist der Einsatz der Sonografie bei Verdacht auf eine Lungenarterienembolie. Rein zentrale Embolien kann man zwar nicht darstellen, meist sind sie jedoch mit Mikroembolisationen in die Peripherie hinein vergesellschaftet. Diese imponieren dann als keilförmige bis runde, echoarme Lungenkonsolidierungen ohne Binnenperfusion ( $\triangle$ Abb. 3). Treten mehr als zwei solcher $0,5-3 \mathrm{~cm}$ großer Läsionen auf, ist eine Lungenembolie sehr wahrscheinlich [2]. Die Läsionen befinden sich häufig dorsobasal.

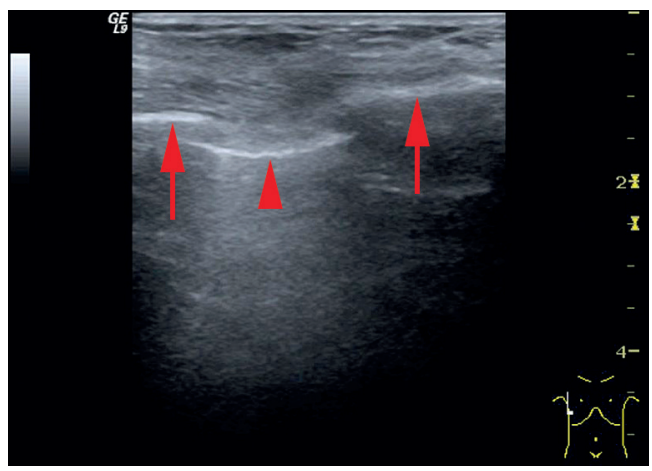

Abb. 2 Rippenreflex mit dorsaler Schallauslöschung (Pfeile), Pleuraecho (Pfeilspitze).

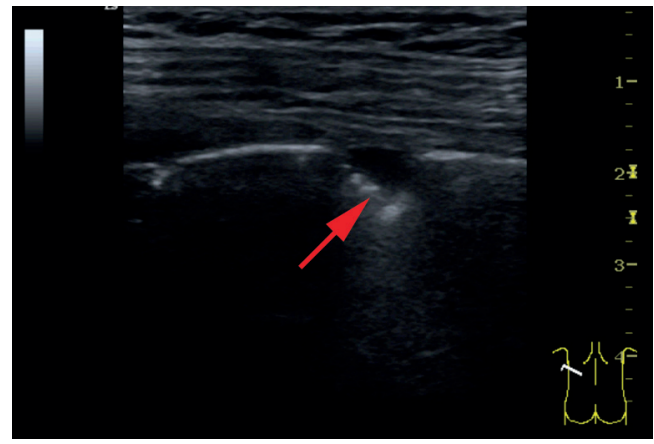

Abb. 3 Echoarme, trianguläre, subpleurale Läsion dorsobasal - typisch für Lungenarterienembolie.

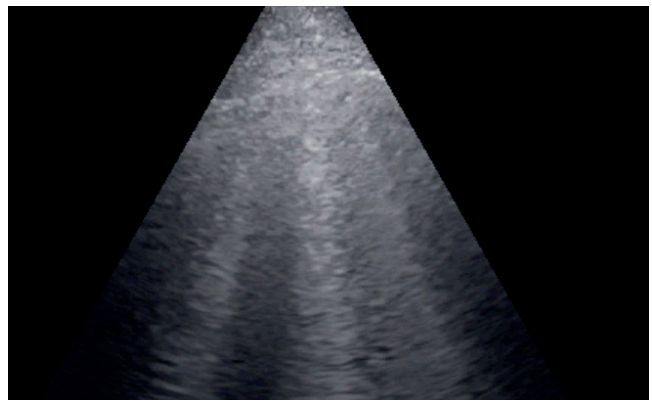

Abb. 4 Vermehrte vertikale, echoreiche B-Linien, hier bei dekompensierter Linksherzinsuffizienz.

Eine Lungenembolie ist hochwahrscheinlich, wenn gleichzeitig eine tiefe Beinvenenthrombose und Zeichen der akuten Rechtsherzbelastung in der fokussierten Echokardiografie auftreten.

Interstitielles Syndrom | Zeigen sich bei zwei oder mehr Anlotpositionen beidseits mind. 3 B-Linien innerhalb eines interkostalen Schallfensters im Longitudinalschnitt, dann spricht man von einem interstitiellen Syndrom ( $\bullet$ Abb. 4). Dies kann verschiedene Ursachen haben, z.B.

- Lungenödem,

- interstitielle Pneumonie,

- Pneumonitis oder

- diffuse Lungenparenchymerkrankungen wie z.B. Lungenfibrose.

COPD | Multimorbide Patienten mit COPD und Luftnot sind eine klinische Herausforderung. In der Notfallsituation kann der Ultraschall helfen, eine dekompensierte Linksherzinsuffizienz von einer (infekt-)exazerbierten COPD zu unterscheiden [3]. 
Abb. 5 Pneumonisches Infiltrat mit einzelnenen, kleinen Aerobronchogrammen (echoreiche Anteile) und großen Fluidobronchogrammen (echoarm bis echofrei). Der Pfeil zeigt auf das Zwerchfell.

Abb. 6 Rippenfraktur mit Unterbrechung des Kortikalisreflexes (Pfeil) und Stufenbildung.

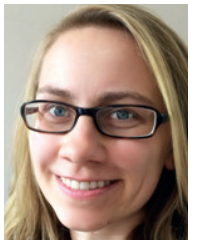

Dr. med. Tanja Kaneko ist Assistenzärztin für Innere Medizin in den Ruppiner Kliniken in Neuruppin. tanjapil@gmx.de

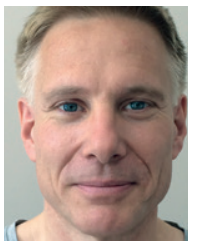

Dr. med. Wolfgang Heinz ist Chefarzt der Inneren Klinik I, Karl-Olga Krankenhaus in Stuttgart. wolfgang.heinz@sana.de

Interessenkonflikt WH gibt an, Vortragshonorare von GE erhalten zu haben. TK gibt an, dass kein Interessenkonflikt besteht.

DOI 10.1055/s-0041-106455 Dtsch Med Wochenschr 2015; 140: 1680-1682

(c) Georg Thieme Verlag KG . Stuttgart · New York . ISSN 0012-0472

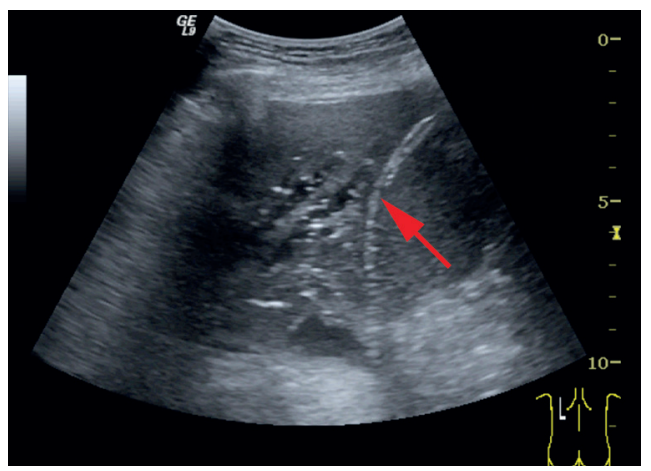

- fehlendes Lungengleiten

- fehlender Lungenpuls

- fehlende B-Linien

- fehlendes Farbzeichen

- vermehrte horizontale Wiederholungsechos

- Nachweis des Lungenpunktes

Ein Hautemphysem an den Anlotstellen schränkt die Untersuchung ein. Daher gelingt die Darstellung der Pleura nicht immer.

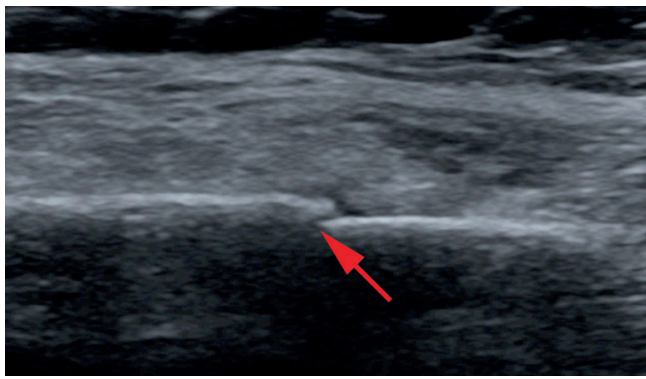

Pleuritis | Atemabhängige Schmerzen finden sich charaktertistischerweise bei einer Pleuritis. Wenn bereits ein pleuritischer Begleiterguss entstanden ist, kann der Auskultationsbefund des Pleurareibens fehlen. Eine reine Pleuritis lässt sich im Röntgen-Thorax nicht erfassen. Sonografisch lassen sich jedoch typische Veränderungen nachweisen. In bis zu 90\% der Fälle imponiert der Pleurareflex aufgeraut und unterbrochen. Mitunter zeigen sich kleinere subpleurale Konsolidierungen und ein schmaler Pleuraerguss.

\section{Leitsymptome Dyspnoe und Fieber}

Lobärpneumonie | Eine Pneumonie ( $\triangle$ Abb. 5) kann sonografisch nie ausgeschlossen werden. Reichen die Infiltrate jedoch bis an die viszerale Pleura, ist Ultraschall ein gutes ergänzendes bildgebendes Verfahren - insbesondere zur Verlaufskontrolle (Einsparen von Strahlenexposition). Typische Zeichen der Lobärpneumonie sind:

- Lungenkonsolidierung („Hepatisation der Lunge“)

- Aerobronchogramm

- Fluidobronchogramm (eher bei poststenotischer Pneumonie)

- ggf. verstärkte Durchblutung im Powerdoppler - vermehrte B-Linien, ausgehend vom schallkopffernen Läsionsrand

Pleuraempyem | Zeigt sich im Zusammenhang mit Dyspnoe und Fieber ein nicht echofreier Pleuraerguss, so sollte differentialdiagnostisch an ein Pleuraempyem gedacht werden. Mittels einer ultraschall-gesteuerten Punktion kann der Verdacht ggf. zeitnah überprüft werden.

\section{Leitsymptom Thoraxschmerz}

Bei Notfällen ist die Sonografie als Primärbildgebung bei der Pneumothoraxdiagnostik mittlerweile „state of the art“. Im Rahmen der FAST-Untersuchung können thorakale Anlotungen leicht und ohne nennenswerte Zeitverzögerung ergänzt werden. Kann man das Lungengleiten darstellen, so ist ein Pneumothorax an der untersuchten Stelle ausgeschlossen. Damit ist ein klinisch relevanter Pneumothorax zum Zeitpunkt der Untersuchung unwahrscheinlich. Zeichen, die für das Vorliegen eines Pneumothorax sprechen, sind:
Frakturen I Im Gegensatz zum konventionellen Röntgenbild bietet Ultraschall die Möglichkeit, ( Abb. 6) zu detektieren. Am Schmerzpunkt weist oft zunächst ein begleitendes echoarmes, subperiostales Hämatom auf die Frakturstelle hin. Der Bruch ist bei genauerer Untersuchung üblicherweise gut anhand der Unterbrechung oder gar Stufenbildung des Kortikalisreflexbandes zu erkennen.

\section{Dokumentation}

M-Mode I Neben den üblichen Dokumentationsmethoden - dem Standbild oder dem Clip - bietet sich insbesondere bei der Pneumothoraxdiagnostik der $\mathrm{M}$-Mode $(\mathrm{M}=$ motion) an. Entlang einer Zeitachse wird die Bewegung einer Bildzeile aufgetragen. Im Normalfall entsteht fernab des Pleuraechos bei anliegender Lunge durch Artefakte ein Rauschen (seashore sign). Fehlt das Lungengleiten, zeigt sich im M-Mode ein starres Bild geprägt von vielen Wiederholungsechos (barcode sign).

Konsequenz für Klinik und Praxis

- Bei Dyspnoe, Fieber und/oder thorakalen Schmerzen sollte man immer eine Ultraschalluntersuchung erwägen.

- Die Sonografie kann bezüglich der weiteren Therapie richtungsweisend sein.

- Die Hauptlimitation der Thoraxsonografie besteht darin, dass Lungenparenchymveränderungen nur dann darstellbar sind, wenn lufthaltige Lunge sie nicht überdeckt.

Vollständiges Literaturverzeichnis unter http://dx.doi.org/10.1055/s-0041-106455 sehr sensitiv Frakturen des knöchernen Thorax 


\section{Literatur}

1 Volpicelli G, Elbarbary M, Blaivas M et al. International evidence-based recommendations for point-of-care lung ultrasound. Intensive Care Med 2012; 38: 577-591

2 Mathis G, Blank W, Reissig A et al. Thoracic ultrasound for diagnosing pulmonary embolism: a prospective multicenter study of 352 patients. Chest 2005; 128: 1531-1538

3 Volpicelli G, Cardinale L, Garofalo G, Veltri A. Usefulness of lung ultrasound in the beside distinction between pulmonary edema and exacerbation of COPD. Emerg Radiol 2008; 15: 145-151 